Article

\title{
Screening of Diatom Strains and Characterization of Cyclotella cryptica as A Potential Fucoxanthin Producer
}

\author{
Bingbing Guo ${ }^{1}$, Bin Liu ${ }^{1}$, Bo Yang ${ }^{1}$, Peipei Sun ${ }^{1}$, Xue Lu ${ }^{1}$, Jin Liu ${ }^{1, *}$ and Feng Chen ${ }^{1,2, *}$ \\ 1 Institute for Food and Bioresource Engineering, College of Engineering, Peking University, Beijing 100871, \\ China; guobb@pku.edu.cn (B.G.); caisanrenju@163.com (B.L.); ly_mikeyang@163.com (B.Y.); \\ zixinsp@163.com (P.S.); gracexuelu@pku.edu.cn (X.L.) \\ 2 South China Sea Bio-Resource Exploitation and Utilization Collaborative Innovation Center, \\ Sun Yat-sen University, Guangzhou 510006, China \\ * Correspondence: gjinliu@pku.edu.cn (J.L.); sfchencoe@pku.edu.cn (F.C.); \\ Tel.: +86-10-627-66391 (J.L.); +86-10-627-45356 (F.C.)
}

Academic Editor: Georg Pohnert

Received: 30 April 2016; Accepted: 29 June 2016; Published: 8 July 2016

\begin{abstract}
Fucoxanthin has been receiving ever-increasing interest due to its broad health beneficial effects. Currently, seaweeds are the predominant source of natural fucoxanthin. However, the disappointingly low fucoxanthin content has impeded their use, driving the exploration of alternative fucoxanthin producers. In the present study, thirteen diatom strains were evaluated with respect to growth and fucoxanthin production potential. Cyclotella cryptica (CCMP 333), which grew well for fucoxanthin production under both photoautotrophic and heterotrophic growth conditions, was selected for further investigation. The supply of nitrate and light individually or in combination were all found to promote growth and fucoxanthin accumulation. When transferring heterotrophic cultures to light, fucoxanthin responded differentially to light intensities and was impaired by higher light intensity with a concomitant increase in diadinoxanthin and diatoxanthin, indicative of the modulation of Diadinoxanthin Cycle to cope with the light stress. Taken together, we, for the first time, performed the screening of diatom strains for fucoxanthin production potential and investigated in detail the effect of nutritional and environmental factors on C. cryptica growth and fucoxanthin accumulation. These results provide valuable implications into future engineering of C. cryptica culture parameters for improved fucoxanthin production and C. cryptica may emerge as a promising microalgal source of fucoxanthin.
\end{abstract}

Keywords: fucoxanthin; Cyclotella cryptica; diatom; heterotrophic cultivation

\section{Introduction}

Fucoxanthin, a specific non-provitamin A carotenoid, is mainly found in brown seaweeds, diatoms and golden algae [1]. Fucoxanthin harbors a very unique structure with an allenic bond, a conjugated carbonyl and a 5,6-monoepoxide [2]. This structure endows fucoxanthin with a variety of biological activities, including anti-obesity and anti-diabetes [3-6], anti-cancer [7-11], anti-allergic [12,13], anti-inflammatory [14,15], anti-oxidation [14], and anti-osteoporotic activities [14]. Both in vitro and in vivo experiments indicated no toxicity of fucoxanthin [16-18].

Currently, fucoxanthin is mainly produced from seaweeds. Nevertheless, there are inherent shortcomings for seaweeds such as slow growth, low fucoxanthin content, insufficient yield, and quality concern due to the severe contamination of ocean by heavy metals [19-24]. By contrast, the marine algae diatoms, which grow rapidly, have high fucoxanthin content, and perform robustly in controlled bioreactors [25-27], are therefore considered as potential alternative producers of 
fucoxanthin. For example, the fucoxanthin content ranges from $0.224 \%$ to $2.167 \%$ of dry weight in diatoms, up to 100 times of that in seaweeds $[28,29]$. Moreover, some diatoms can grow in the dark using sugar as the only energy and carbon resource [30]. It has been reported that heterotrophic Nitzschia laevis grew well and reached high cell density [31], indicative of the potential of diatom fermentation for industrial production.

The biosynthetic pathway of fucoxanthin remains unclear in diatoms [32,33]. It has been proposed that fucoxanthin can be either from neoxanthin or from diadinoxanthin (Figure 1). Violaxanthin is the precursor of neoxanthin, together with zeaxanthin and antheraxanthin constituting Violaxanthin Cycle. This cycle serves as a balanced cycle responding to a wide range of light intensity variation [32,34]. Upon high light, the algal cell tends to accumulate zeaxanthin at the expense of violaxanthin, leading to low level of neoxanthin and fucoxanthin as well. Diadinoxanthin Cycle, which consists of diadinoxanthin and diatoxanthin [35], serves the most important short-term photoprotective mechanism [36,37]. For example, when light intensity increases, diadinoxanthin tends to be converted to diatoxanthin for self-protection, leading to reduced fucoxanthin biosynthesis [38-40].

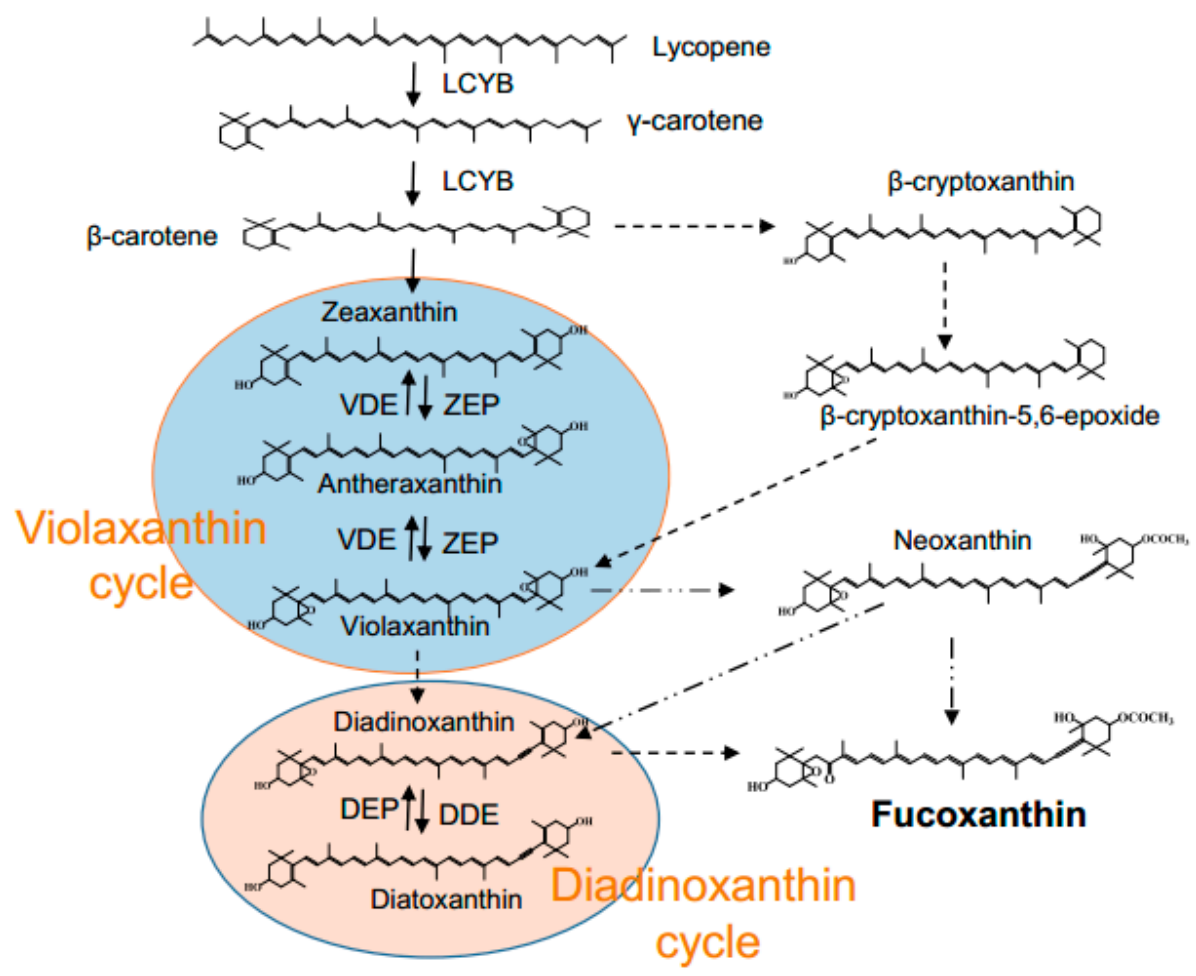

Figure 1. Proposed fucoxanthin biosynthetic steps in diatoms. LCYB: lycopene $\beta$-cyclase; VDE: violaxanthin de-epoxidases; ZEP: zeaxanthin epoxidases; DEP: diatoxanthin epoxidases; DDE: diadinoxanthin de-epoxidases. Solid arrows indicate steps that have been validated, while dotted arrows designate the steps to be defined.

Growth and fucoxanthin content of diatoms may vary considerably across species and/or strains and are dependent on culture conditions. Therefore, it is imperative to employ a high performance diatom strain along with an optimal operational protocol for fucoxanthin production. There are only a limited number of reports about diatoms for fucoxanthin, with the focus on photoautotrophic growth and exploration of fucoxanthin extraction methods [2,28,41-43]. The utilization of heterotrophic growth of diatoms for fucoxanthin has been rarely touched $[31,37,44]$. The disappointingly low fucoxanthin production remains a large barrier for using diatoms as a potential source alternative to seaweeds. Comprehensive strain screening for fucoxanthin-rich producer and rational culture optimization represent feasible directions toward enhanced fucoxanthin production by diatoms, which 
to our best knowledge, have not been done. Hence, the main objective of the present study is to select a good strain capable of growing well both photoautotrophically and heterotrophically and optimize several key biological and engineering parameters for improved fucoxanthin production under laboratory conditions. To this end, we screened 13 naturally occurring diatom strains from several culture collection centers and identified a promising strain, Cyclotella cryptica (CCMP 333), with regard to growth and fucoxanthin production. This strain was investigated in detail in flasks to evaluate the effect of nutritional and environmental factors on growth and fucoxanthin accumulation. These results provide valuable implications into future exploration of $C$. cryptica for possible production of fucoxanthin.

\section{Results}

\subsection{Fucoxanthin Production of Diatoms under Photoautotrophic Conditions}

The fucoxanthin production potential of thirteen diatoms was first evaluated under photoautotrophic conditions with the light intensity of $30 \mu \mathrm{mol} \cdot \mathrm{m}^{-2} \cdot \mathrm{s}^{-1}$. As indicated by Figure 2, most of the strains tested had a fucoxanthin content of over $0.8 \%$ of dry weight, and O. aurita was the strain with highest fucoxanthin content (1.50\%). By contrast, Thalassiosira pseudonana (CCMP 1335) exhibited the lowest fucoxanthin content (below $0.2 \%$ ). As for fucoxanthin productivity, nine of the thirteen strains had a value above $0.1 \mathrm{mg} \cdot \mathrm{L}^{-1} \cdot \mathrm{d}^{-1}$, with Odontella aurita (CCMP 1796) and Phaeodactylum tricornutum (UTEX 646) being the greatest. By contrast, Thalassiosira weissflogii (CCMP 1051) and T. pseudonana had the lowest fucoxanthin productivity, which were less than $0.05 \mathrm{mg} \cdot \mathrm{L}^{-1} \cdot \mathrm{d}^{-1}$.

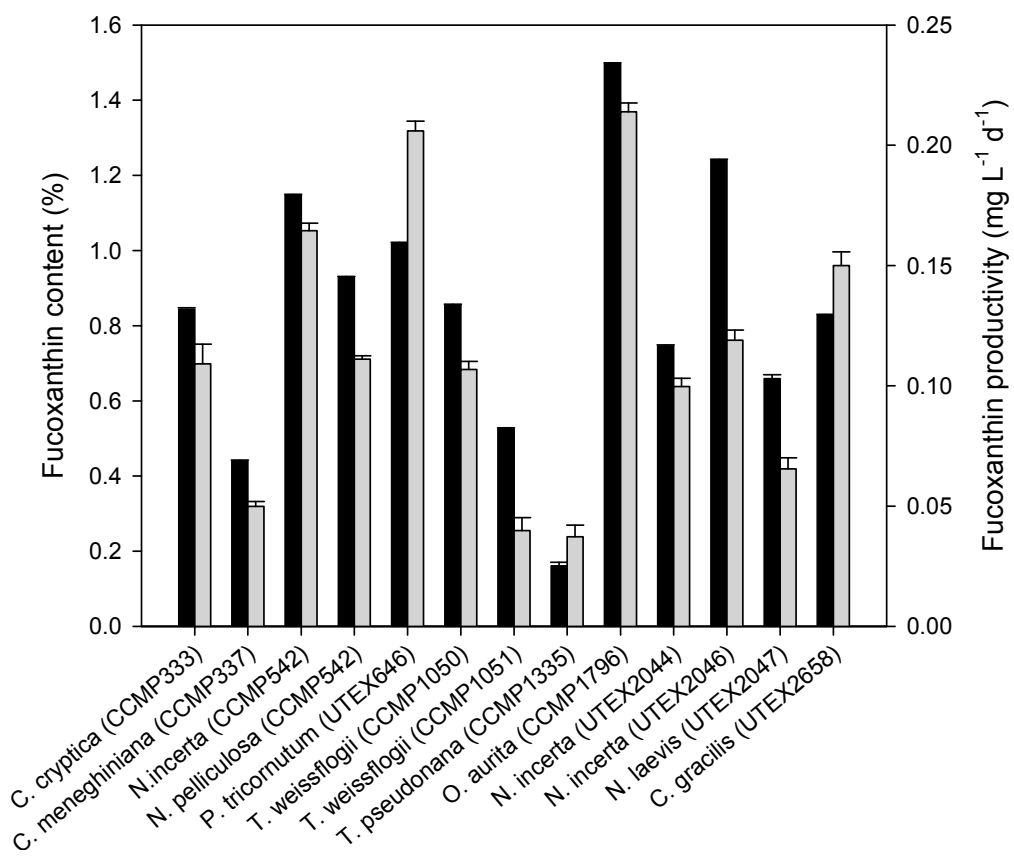

Figure 2. Fucoxanthin content and productivity of thirteen diatom strains under photoautotrophic conditions. Cells on Day 14 were harvested for analysis. Dark bars represent the fucoxanthin content and grey ones represent the fucoxanthin productivity. The fucoxanthin productivity was expressed as fucoxanthin yield divided by culture days. Data are means of three replicates and error bars indicate standard deviations.

\subsection{Growth and Fucoxanthin Production Potential of Diatoms under Heterotrophic Conditions}

Of the thirteen diatoms tested, only six could grow on glucose without light, namely, Cyclotella cryptica (CCMP 333), Cyclotella meneghiniana, Navicula incerta (CCMP 542), Navicula incerta (UTEX 2044), Navicular incerta (UTEX 2046) and Nitzschia laevis (UTEX 2047), though the biomass 
concentration achieved was relatively low (Figure 3). Comparatively, C. cryptica had a higher biomass concentration than the other five strains. In addition, C. cryptica had the highest fucoxanthin content, which was $0.77 \%$ of dry weight and comparable to that under photoautotrophic conditions. Interestingly, C. meneghiniana had a higher fucoxanthin content under heterotrophic growth conditions than under photoautotrophic conditions. On the contrary, the other four strains showed a decrease in fucoxanthin content under heterotrophic growth conditions compared to under photoautotrophic conditions. Taken together, C. cryptica grew relatively better among the six strains under heterotrophic growth conditions and its fucoxanthin content was comparable to that of other diatom strains under photoautotrophic conditions, thus it was chosen for the subsequent investigation.

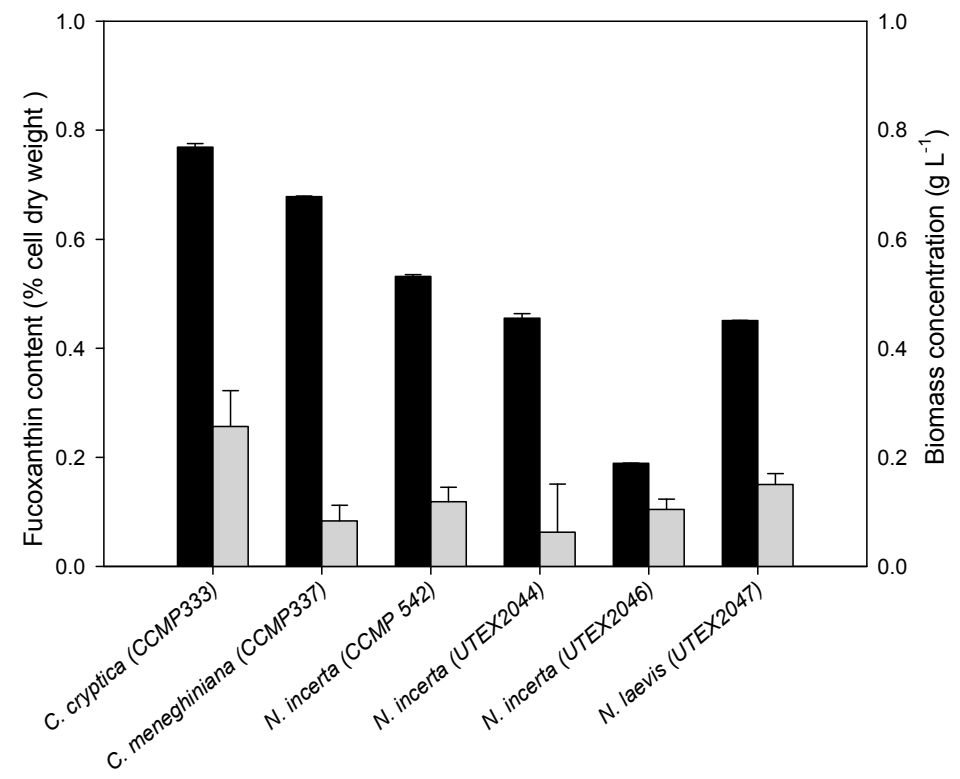

Figure 3. Fucoxanthin content (black column) and biomass concentration (gray column) of diatom strains capable of growing on glucose without light. Cells on Day 6 were collected for analysis. The solid black bar and grey bar represent the fucoxanthin content and biomass concentration, respectively. Data are means of three replicates and error bars indicate standard deviations.

\subsection{Effect of Nitrate and/or Light Supply on Growth and Fucoxanthin Production of C. cryptica}

We found that C. cryptica grew better in modified SK medium than in F/2 medium under heterotrophic conditions (data not shown). Based on the modified SK medium, we further tested the effect of nitrate and/or light supply on growth and fucoxanthin production of C. cryptica (Figure 4a,b). Nitrate supply showed almost no effect on C. cryptica growth before entering the stationary phase (Day 6); afterwards, the algal cells showed a decline in biomass concentration for the control cultures while a slight increase for the nitrate-supplied cultures (Figure 4a). Consequently, the final biomass concentration reached $1.15 \mathrm{~g} \cdot \mathrm{L}^{-1}$ for the nitrate-supplied cultures, $26.4 \%$ higher than the control. By contrast, light supply had a more significantly positive effect on cell growth and led to a greater biomass concentration than the control during the whole culture period. Noteworthy, similar to the control, the light-supplied cells reached the highest biomass concentration $\left(1.30 \mathrm{~g} \cdot \mathrm{L}^{-1}\right)$ on Day 5 , followed by a gradual decline. The supply of both nitrate and light further promoted the cell growth and biomass concentration reached the maximum of $1.72 \mathrm{~g} \cdot \mathrm{L}^{-1}$ on Day 6, which was $55.0 \%$ higher than that of the control cultures.

The effect of nitrate and/or light supply on fucoxanthin content is shown in Figure $4 \mathrm{~b}$. The control cultures maintained a relatively stable fucoxanthin content. Nitrate supply benefited the accumulation of fucoxanthin, which increased rapidly after Day 2 and reached the maximum of 1.18\% on Day 6, $75.4 \%$ higher than that of the control. Light supply had a similar effect to nitrate supply and promoted 
fucoxanthin accumulation, with the maximum content of $0.89 \%$ of dry weight achieved on Day 6 . By contrast, the supply of both nitrate and light promoted fucoxanthin accumulation more significantly, which occurred on Day 2 and then increased slightly to the maximum content of $1.29 \%$ of dry weight on Day 4, 86.0\% higher than the maximum of control on Day 6. Interestingly, under all tested conditions, fucoxanthin content increased as the cells grew and started to decline once entering the stationary growth phase, indicating that fucoxanthin accumulation is possibly growth dependent.
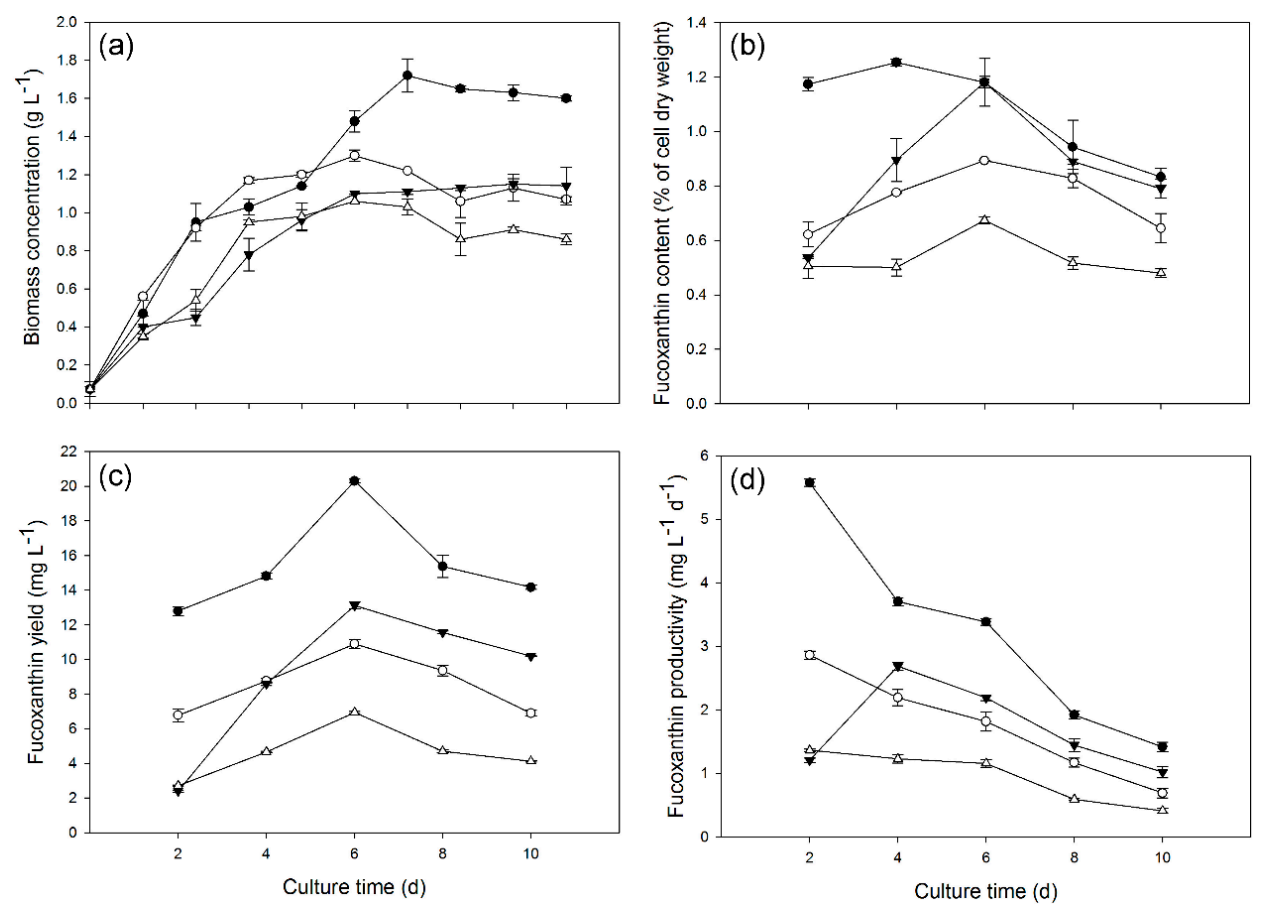

Figure 4. Biomass concentration (a); Fucoxanthin contents (b); Fucoxanthin yield (c); and Fucoxanthin productivity (d) of $C$. cryptica. Solid circle and empty circle represent the culture with or without nitrate in the light, and solid triangle down and empty triangle up represent the culture with or without nitrate under dark, respectively. The nitrate concentration is $1.00 \mathrm{~g} \cdot \mathrm{L}^{-1}$ and the light intensity is $30 \mu \mathrm{mol} \cdot \mathrm{m}^{-2} \cdot \mathrm{s}^{-1}$. Data are means of three replicates and error bars indicate standard deviations.

Both nitrate and light supply independently promoted fucoxanthin yield considerably (Figure 4c). The combination of nitrate and light supply further increased fucoxanthin yield. Regardless of the culture conditions, the maximum fucoxanthin yield was achieved on Day 6. Specifically, the maximum fucoxanthin yield under the supply of both nitrate and light was $20.30 \mathrm{mg} \cdot \mathrm{L}^{-1}, 192.5 \%$ higher than that of the control. As for the productivity, the supply of nitrate and light individually or in combination all promoted it, with the combination of nitrate and light being most effective (Figure $4 \mathrm{~d}$ ). Clearly, fucoxanthin productivity remained relatively high in early growth days and declined gradually, regardless of culture conditions. This may indicate that $C$. cryptica in exponential growth phase is preferred for fucoxanthin production.

\subsection{Effect of Light Intensity on Growth and Fucoxanthin Production}

We demonstrated that light supply affected the growth and fucoxanthin production of C. cryptica. To further investigate the effect of light intensities, four light intensities of 10, 20, 30, and $40 \mu \mathrm{mol} \cdot \mathrm{m}^{-2} \cdot \mathrm{s}^{-1}$ were applied (Figure 5). The C. cryptica cells were first grown without light and then subjected to different light intensities, using the dark-grown cells as the control. Obviously, light intensity had no significant effect on cell growth under this tested conditions (Figure 5a). The cells began to decline after three days of cultivation (Figure 5a), probably as a result of nutrition depletion. By contrast, light intensities influenced the cellular fucoxanthin content differentially: light intensities 
between 10 and $30 \mu \mathrm{mol} \cdot \mathrm{m}^{-2} \cdot \mathrm{s}^{-1}$ promoted the fucoxanthin accumulation while $40 \mu \mathrm{mol} \cdot \mathrm{m}^{-2} \cdot \mathrm{s}^{-1}$ had no beneficial effect (Figure 5b). Quantitatively, on Day 1, the fucoxanthin content was $1.08 \%$ of dry weight under the light intensity of $10 \mu \mathrm{mol} \cdot \mathrm{m}^{-2} \cdot \mathrm{s}^{-1}, 33.3 \%$ higher than of the control $(0.76 \%)$, while the fucoxanthin content under the light intensity of $40 \mu \mathrm{mol} \cdot \mathrm{m}^{-2} \cdot \mathrm{s}^{-1}$ was $0.62 \%$, lower than that of the control. The effect of light intensity on fucoxanthin yield was also examined (Figure 5c). Overall, low light intensities of $10-30 \mu \mathrm{mol} \cdot \mathrm{m}^{-2} \cdot \mathrm{s}^{-1}$ were better than the relatively high light intensity of $40 \mu \mathrm{mol} \cdot \mathrm{m}^{-2} \cdot \mathrm{s}^{-1}$.
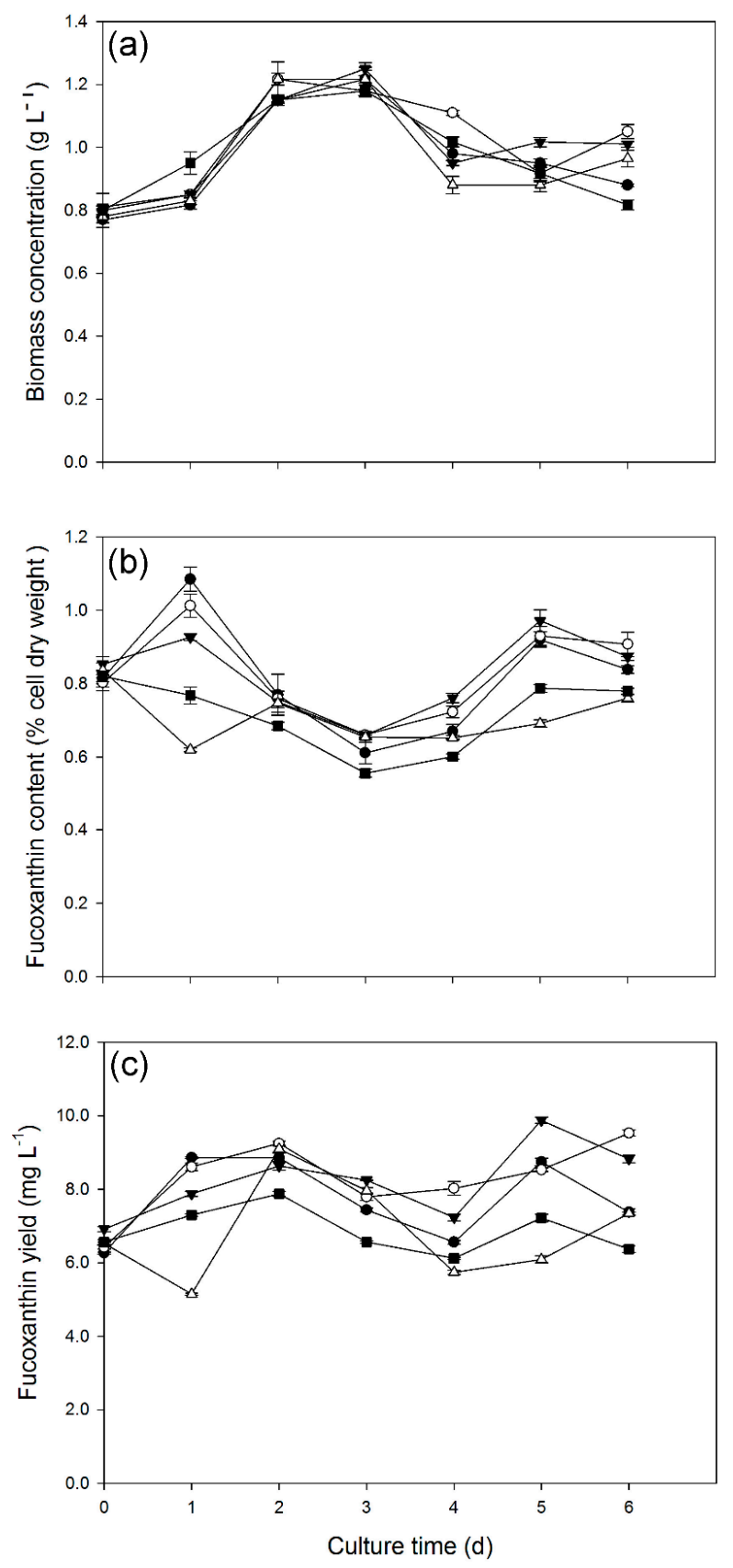

Figure 5. Biomass concentration (a); Fucoxanthin contents (b); and Fucoxanthin yield (c) under different light intensity. Solid circle, empty cycle, solid triangle down and empty triangle up represent an illumination intensity condition of $10,20,30$, and $40 \mu \mathrm{mol} \cdot \mathrm{m}^{-2} \cdot \mathrm{s}^{-1}$, respectively, with solid square as the dark-growing control. Data are means of three replicates and error bars indicate standard deviations. 


\subsection{Effect of Light Intensity on Diadinoxanthin and Diatoxanthin}

HPLC analysis of the carotenoid composition of C. cryptica extracts found two unknown major peaks right after fucoxanthin (Figure 6). Fucoxanthin was identified by comparing the retention time of a fucoxanthin standard (Figure 6a,b), and was further confirmed by its mass spectrometry (Figure 6c) and UV-visible spectrum ( $\lambda_{\max }$ at $449.8 \mathrm{~nm}$ ) (Figure $6 \mathrm{~d}$ ). To identify unknown peaks 2 and 3, mass spectrometry analysis was performed. Through the comparison of their fragment patterns with previous reports $[45,46]$, they were annotated to be diadinoxanthin and diatoxanthin, respectively (Table 1).

Clearly, as the light intensity increased from 10 to $30 \mu \mathrm{mol} \cdot \mathrm{m}^{-2} \cdot \mathrm{s}^{-1}$, diadinoxanthin and diatoxanthin increased while fucoxanthin declined on Day 1 (Figure 7). When the light intensity further reached to $40 \mu \mathrm{mol} \cdot \mathrm{m}^{-2} \cdot \mathrm{s}^{-1}$, diadinoxanthin and diatoxanthin remained relatively stable; by contrast, fucoxanthin dropped sharply. This similar pattern was also observed on Day 3 and Day 5.

Table 1. Compounds tentatively assigned in the crude extracts of C. cryptica.

\begin{tabular}{|c|c|c|c|c|c|}
\hline Peak No. & Compound & $t_{r} *(\min )$ & $\lambda_{\max }(\mathrm{nm})$ & Ionization Type & $\begin{array}{l}\text { Mass Spectra } \mathbf{M}^{+} m / z \\
\text { (Relative Intensity, \%) }\end{array}$ \\
\hline 2 & Diadinoxanthin & 21.059 & $\begin{array}{l}444.9 \\
475.3\end{array}$ & $\begin{array}{c}{[\mathrm{M}+\mathrm{H}]^{+}} \\
{\left[\mathrm{M}+\mathrm{H}-\mathrm{H}_{2} \mathrm{O}\right]^{+}}\end{array}$ & $\begin{array}{c}565.4(10) ; 583.4(100) ; \\
584.4(50) ; 585.4(10)\end{array}$ \\
\hline 3 & Diatoxanthin & 26.257 & $\begin{array}{l}449.8 \\
478.9\end{array}$ & $\begin{array}{c}{[\mathrm{M}+\mathrm{H}]^{+}} \\
{\left[\mathrm{M}+\mathrm{H}-\mathrm{H}_{2} \mathrm{O}\right]^{+}}\end{array}$ & $\begin{array}{c}549.4(10) ; 567.4(100) ; \\
568.4(50) ; 569.4(10)\end{array}$ \\
\hline
\end{tabular}
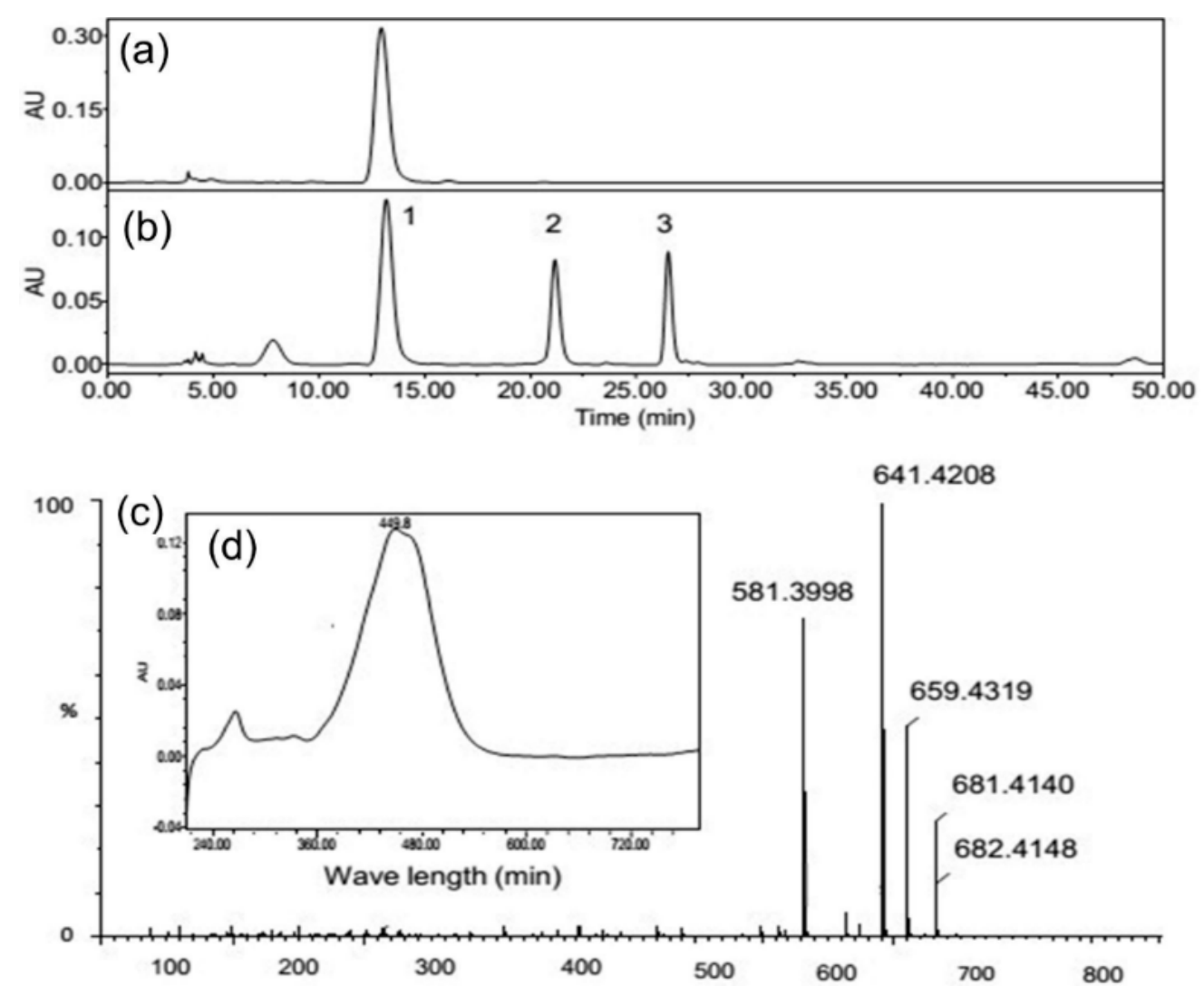

Figure 6. HPLC (High Performance Liquid Chromatography) chromatograms of fucoxanthin standard (a) and the crude extract of C. cryptica (b). Mass spectroscopy (c) of peak 1 in (b) and its visible absorption spectra (d). Absorbance in (a) and (b) was recorded at $449 \mathrm{~nm}$. Peaks were identified as follows: 1, fucoxanthin; 2, diadinoxanthin; and 3, diatoxanthin. 


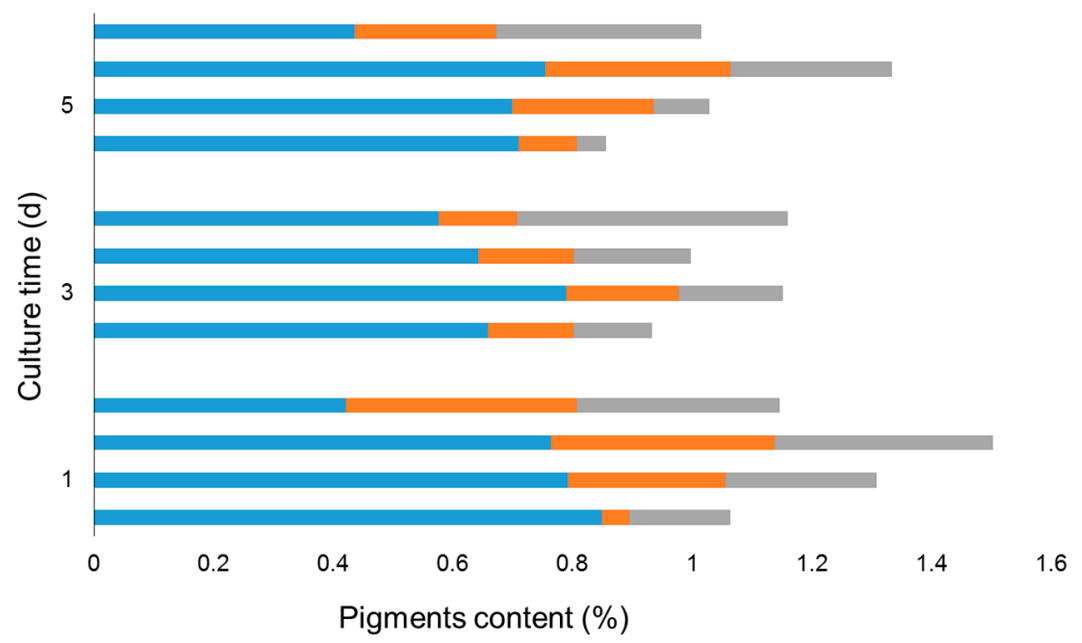

Figure 7. Fucoxanthin content (blue bar), Diadinoxanthin content (orange bar) and Diatoxanthin content (grey bar) under different light intensity. Bars from the bottom up in every group represent the light intensity of $10,20,30$, and $40 \mu \mathrm{mol} \cdot \mathrm{m}^{-2} \cdot \mathrm{s}^{-1}$, respectively. Diadinoxanthin and diatoxanthin contents were quantified as fucoxanthin equivalents with the same method.

\section{Discussion}

The growth and fucoxanthin synthesis of diatoms have been previously reported under photoautotrophic conditions [2,42]. Heterotrophic cultivation of diatoms has also been documented, but not for fucoxanthin production $[47,48]$. In the present study, we for the first time performed a screening study using thirteen diatom strains across ten species, with respect to their growth and fucoxanthin accumulation under both photoautotrophic and heterotrophic conditions (Figures 2 and 3). C. cryptica (CCMP 333) was selected because it could grow heterotrophically on glucose better and accumulated more fucoxanthin than other tested diatom strains (Figure 3). Sugars, glucose in particular, are the commonly used organic carbon source for the fermentation of microorganisms including algae [31,49-51]. Algae fermentation has some significant advantages and thus has been considered as a promising production strategy for value-added products [31,49,52]. Nevertheless, the biomass concentration achieved in our study was relatively low (Figure 3). We replaced F/2 medium with modified SK medium, which was derived from Pahl et al. [37] with minor modifications, and observed a considerable increase in biomass concentration (Figures 2-4).

Nitrogen is a primary nutrient for algae growth. There are multiple nitrogen sources such as organic nitrogen from tryptone, yeast extract and amino acids, and inorganic nitrogen from nitrate, ammonium and urea. The preference of algae for nitrogen sources is species and/or strain dependent [53-58]. The modified SK medium used in the present study contains the organic nitrogen of tryptone and yeast extract. We found that nitrate supply to modified SK medium benefited C. cryptica growth leading to a higher biomass concentration (Figure 4). This was in line with previous studies that high level of available nitrogen promoted biomass accumulation of diatoms [20] as well as of other algae strains $[57,58]$. Interestingly, the higher biomass concentration caused by nitrate supply only occurred in the stationary growth phase (Figure 4a), which may indicate that the nitrogen level of modified SK medium is not sufficient for $C$. cryptica growth under our tested conditions. Nitrogen also plays an important role in regulating cellular metabolites. Nitrate needs to be converted to nitrite and then to ammonium for assimilation by algae. Ammonium is used to synthesize glutamate, which is a precursor for the biosynthesis of chlorophyll for photosynthesis process [28]. Nitrate supply might upregulate the biosynthesis of chlorophyll and, consequently, promote fucoxanthin accumulation (Figure $4 b$ ), as fucoxanthin is a core part of photosystem in diatoms. Consistent with this, downregulation of glutamate was observed upon nitrogen deprivation, accompanied by a decrease in chlorophyll and fucoxanthin contents [59]. 
Light has dual effects on fucoxanthin biosynthesis: light benefits fucoxanthin accumulation when in low intensities, but impairs fucoxanthin when intensity exceeds a certain value, say $40 \mu \mathrm{mol} \cdot \mathrm{m}^{-2} \cdot \mathrm{s}^{-1}$ in our study (Figure 5), in line with the previous reports that algal fucoxanthin content was higher under low light intensity $[60,61]$. Thus, higher fucoxanthin production was obtained under low light intensities (Figure 5c). The beneficial effect of low light intensity on fucoxanthin content and production was also observed in previous reports [33,42]. We hypothesize that the fucoxanthin variation caused by light may be related to the modulation of Diadinoxanthin Cycle [36,37]. Under high light intensities, to cope with the abiotic stress, diatom cells tend to convert diadinoxanthin to diatoxanthin at the expense of fucoxanthin, leading to reduced fucoxanthin biosynthesis (Figure 1).

In addition to nitrogen and light, there are many other factors worth of investigation for growth and fucoxanthin production of C. cryptica, including different medium and agitation conditions [62]. Our study highlights the production potential of C. cryptica, but its biomass concentration is still relatively low. Nevertheless, our study represents a pioneering effect of using heterotrophic diatom cultures for fucoxanthin. The future exploration of this alga for fucoxanthin production may lie in the optimization of heterotrophic parameters for improving the glucose-to-biomass conversion efficiency and cell density and the development of rational illumination of heterotrophic cultures for maintaining fucoxanthin content.

\section{Materials and Methods}

\subsection{Strains and Culture Conditions}

Thirteen diatom strains were used, including C. cryptica (CCMP 333), C. meneghiniana (CCMP 337), N. incerta (CCMP 542), N. pelliculosa (CCMP 543), T. weissflogii (CCMP 1050), T. weissflogii (CCMP 1051), T. pseudonana (CCMP 1335), O. aurita (CCMP 1796), P. tricornutum (UTEX 646), N. incerta (UTEX 2044), N. incerta (UTEX 2046), N. laevis (UTEX 2047), and Ch. gracilis (UTEX 2658). They were obtained from National Center for Marine Algae and Microbiota (NCMA) or Culture Collection of Algae at The University of Texas at Austin (UTEX).

For the screening experiment, all these strains were cultivated in $250 \mathrm{~mL}$ Erlenmeyer flasks containing $50 \mathrm{~mL}$ of sterile $\mathrm{F} / 2$ medium (recipe from NCMA) under an illumination intensity of $30 \mu \mathrm{mol} \cdot \mathrm{m}^{-2} \cdot \mathrm{s}^{-1}$ till the exponential stage. Alga cells were then inoculated at $10 \%(\mathrm{v} / \mathrm{v})$ into $1000 \mathrm{~mL}$ conical flasks containing $400 \mathrm{~mL}$ of the same medium with a salinity of $27.5 \%$ and grown at $25^{\circ} \mathrm{C}$ for 14 days. For photoautotrophic cultures, the illumination intensity was $30 \mu \mathrm{mol} \cdot \mathrm{m}^{-2} \cdot \mathrm{s}^{-1}$ with a light/dark regime of $12 \mathrm{~h} / 12 \mathrm{~h}$. For heterotrophic cultures, ten grams of glucose were added per liter of medium. All medium was sterilized at $121^{\circ} \mathrm{C}$ for $20 \mathrm{~min}$. Alga cells were harvested through centrifugation and then freeze-dried for $48 \mathrm{~h}$ before being used to extract pigments.

For the growing experiment of $C$. cryptica, modified SK medium was used with modifications reported by Pahl et al. [54]. The base medium consists of (per liter) $22.5 \mathrm{~g}$ sea salt (Sigma, St. Louis, MO,

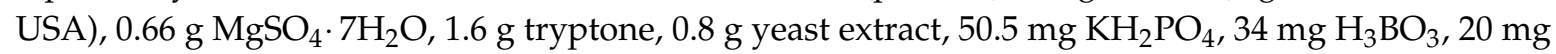
$\mathrm{FeSO}_{4} \cdot 7 \mathrm{H}_{2} \mathrm{O}, 4.3 \mathrm{mg} \mathrm{MnCl} 2 \cdot 4 \mathrm{H}_{2} \mathrm{O}, 0.3 \mathrm{mg} \mathrm{ZnCl}, 0.13 \mathrm{mg} \mathrm{CoCl} \cdot 6 \mathrm{H}_{2} \mathrm{O}$ and $0.03 \mathrm{mg} \mathrm{Na} \mathrm{MoO}_{4} \cdot 2 \mathrm{H}_{2} \mathrm{O}$, and was supplemented with $10 \mathrm{~g} \cdot \mathrm{L}^{-1}$ glucose and $480 \mathrm{mg} \cdot \mathrm{L}^{-1} \mathrm{Na}_{2} \mathrm{SiO}_{3} \cdot 5 \mathrm{H}_{2} \mathrm{O}$. The $\mathrm{pH}$ of the medium was adjusted to 7.5 prior to autoclaving at $121^{\circ} \mathrm{C}$ for $20 \mathrm{~min}$.

For the nitrate-induced experiment of $C$. cryptica, $1.00 \mathrm{~g} \cdot \mathrm{L}^{-1}$ sodium nitrate was added in the base medium before sterilization, while base medium without supplement of sodium nitrate was used as the control. Both of the two groups were cultured in continuous light $\left(30 \mu \mathrm{mol} \cdot \mathrm{m}^{-2} \cdot \mathrm{s}^{-1}\right)$ and dark conditions with an orbital shaking of $150 \mathrm{rpm}$ at $25^{\circ} \mathrm{C}$, respectively. For the dark-light experiment, the culture was cultivated for 4 days to reach the exponential phase in dark and then exposed to different continuous light intensities, 10, 20, 30, or $40 \mu \mathrm{mol} \cdot \mathrm{m}^{-2} \cdot \mathrm{s}^{-1}$. 


\subsection{Determination of Biomass Concentration and Fucoxanthin Production}

Samples were measured and the biomass concentration was calculated as previously described [63]. Briefly, five milliliters of cell suspension were sampled to determine the biomass concentration. Cells were washed for two times with distilled water before being filtered on a pre-weighted Whatman GF/C filter (Cat No. 1822-047). The filters containing the algae were dried in a vacuum oven at $80^{\circ} \mathrm{C}$ for $24 \mathrm{~h}$ and was subsequently cooled down to room temperature for weighing. The biomass concentration is expressed as cells dry weight per liter.

The maximum specific growth rate $\left(\mu_{\max }\right)$ was calculated according to:

$$
\mu_{\max }=\left(\ln X_{2}-\ln X_{1}\right) /\left(t_{2}-t_{1}\right)
$$

where $X_{2}$ and $X_{1}$ are the dry weight $\left(\mathrm{g} \cdot \mathrm{L}^{-1}\right)$ at time $t_{2}$ and $t_{1}$, respectively.

The fucoxanthin yield was the result of fucoxanthin content multiplied by biomass concentration, while the fucoxanthin productivity was expressed as fucoxanthin yield divided by culture days.

\subsection{Pigments Extraction}

For all extraction procedures, $20 \mathrm{mg}$ of lyophilized cell powder was ground and then extracted with $3 \mathrm{~mL}$ analytical ethanol under dim light. After shaking for $1 \mathrm{~h}$ at room temperature, the whole solutions carrying grinded cells were centrifuged at $3000 \mathrm{r} / \mathrm{min}$ for $5 \mathrm{~min}$. The supernatant was collected and the pellets were used to extract pigments for another two times under the same conditions. All three supernatants were combined and evaporated with $\mathrm{N}_{2}$ in dim light. Subsequently, crude pigments were dissolved in pure ethanol and filtered through a $0.22 \mu \mathrm{m}$ filter (Anpel, Shanghai, China) prior to HPLC and LC-MS analysis.

\subsection{Analysis of Pigments by HPLC and LC-MS}

Fucoxanthin was detected using Waters 2695 HPLC system (Waters, Milford, MA, USA) with a PDA detector and a $C_{18}$ reverse phase bar $(5 \mathrm{~mm}$ particle size, $250 \mathrm{~mm} \times 4.6 \mathrm{~mm}$ ID). Specific analysis method was set according to Kim et al. [2] with minor modifications. Briefly, the mobile phase consisted of methanol and water with a flow rate of $0.8 \mathrm{~mL} \cdot \mathrm{min}^{-1}$. In the gradient condition, methanol/water ratio was increased from 90:10 to 100:0 in $30 \mathrm{~min}$ and then the 100\% methanol flow held for another $20 \mathrm{~min}$. The chromatogram was recorded at $449 \mathrm{~nm}$. The fucoxanthin standards were used for the construction of calibration curve at a concentration range of $10-250 \mu \mathrm{g} \cdot \mathrm{mL}^{-1}$. Pigments were also confirmed by liquid chromatography-mass spectrometry (LC-MS, Waters Xevo G2QTOF, Milford, MA, USA) with an atmospheric pressure chemical ionization (APCI) using the same chromatographic column under the same mobile phase conditions.

\subsection{Statistical Analysis}

All cultivations were performed at least in duplicate. All determinative data were collected from triplicate samples and the final values were expressed as mean value \pm standard deviation. Statistical significance was evaluated by ANOVA and $t$-test using SPSS programs (Version 19.0, IBM SPSS, Chicago, IL, USA) at a level of $P<0.05$.

\section{Conclusions}

In summary, we for the first time screened thirteen diatom strains with respect to their growth and fucoxanthin accumulation, and selected C. cryptica as a promising producer for fucoxanthin. Detailed investigation revealed that the supply of nitrate and light individually or in combination promoted growth and fucoxanthin accumulation of C. cryptica. When transferring heterotrophic algal cultures to light, fucoxanthin responded differentially to light intensities by regulating the Diadinoxanthin Cycle and was upregulated by low intensities. Our study represents a research starting point and 
provides valuable implications into future engineering of culture parameters for improved fucoxanthin production by C. cryptica.

Acknowledgments: This research was financially supported by Public Science and Technology Research Funds Projects of Ocean (Project 201505032), and partly supported by National Natural Science Foundation of China (Projects 31471717 and 31571807).

Author Contributions: B.G. and B.L. conceived and designed the experiments; B.G., P.S. and X.L. performed the experiments; B.G., J.L. and B.Y. analyzed the data; and B.G., J.L. and F.C. wrote and revised the paper.

Conflicts of Interest: The authors declare no conflict of interest.

\section{References}

1. Fung, A.; Hamid, N.; Lu, J. Fucoxanthin content and antioxidant properties of Undaria pinnatifida. Food Chem. 2013, 136, 1055-1062. [CrossRef] [PubMed]

2. Kim, S.M.; Jung, Y.J.; Kwon, O.N.; Cha, K.H.; Um, B.H.; Chung, D.; Pan, C.H. A potential commercial source of fucoxanthin extracted from the microalga Phaeodactylum tricornutum. Appl. Biochem. Biotechnol. 2012, 166, 1843-1855. [CrossRef] [PubMed]

3. Maeda, H.; Hosokawa, M.; Sashima, T.; Murakami-Funayama, K.; Miyashita, K. Anti-obesity and anti-diabetic effects of fucoxanthin on diet-induced obesity conditions in a murine model. Mol. Med. Rep. 2009, 2, 897-902. [CrossRef] [PubMed]

4. Maeda, H.; Hosokawa, M.; Sashima, T.; Funayama, K.; Miyashita, K. Effect of medium-chain triacylglycerols on anti-obesity effect of fucoxanthin. J. Oleo Sci. 2007, 56, 615-621. [CrossRef] [PubMed]

5. Maeda, H.; Hosokawa, M.; Sashima, T.; Takahashi, N.; Kawada, T.; Miyashita, K. Fucoxanthin and its metabolite, fucoxanthinol, suppress adipocyte differentiation in 3T3-L1 cells. Int. J. Mol. Med. 2006, 18, 147-152. [CrossRef] [PubMed]

6. Peng, J.; Yuan, J.P.; Wu, C.F.; Wang, J.H. Fucoxanthin, a marine carotenoid present in brown seaweeds and diatoms: Metabolism and bioactivities relevant to human health. Mar. Drugs 2011, 9, 1806-1828. [CrossRef] [PubMed]

7. Lee, J.C.; Hou, M.F.; Huang, H.W.; Chang, F.R.; Yeh, C.C.; Tang, J.Y.; Chang, H.W. Marine algal natural products with anti-oxidative, anti-inflammatory, and anti-cancer properties. Cancer Cell Int. 2013, 13, 55. [CrossRef] [PubMed]

8. Satomi, Y. Fucoxanthin induces GADD45A expression and G1 arrest with SAPK/JNK activation in LNCap human prostate cancer cells. Anticancer Res. 2012, 32, 807-813. [PubMed]

9. Rokkaku, T.; Kimura, R.; Ishikawa, C.; Yasumoto, T.; Senba, M.; Kanaya, F.; Mori, N. Anticancer effects of marine carotenoids, fucoxanthin and its deacetylated product, fucoxanthinol, on osteosarcoma. Int. J. Oncol. 2013, 43, 1176-1186. [CrossRef] [PubMed]

10. Kim, K.N.; Ahn, G.; Heo, S.J.; Kang, S.M.; Kang, M.C.; Yang, H.M.; Kim, D.; Roh, S.W.; Kim, S.K.; Jeon, B.T. Inhibition of tumor growth in vitro and in vivo by fucoxanthin against melanoma B16F10 cells. Environ. Toxicol. Pharmacol. 2013, 35, 39-46. [CrossRef] [PubMed]

11. Das, S.K.; Hashimoto, T.; Kanazawa, K. Growth inhibition of human hepatic carcinoma HepG2 cells by fucoxanthin is associated with down-regulation of cyclin D. BBA Gen. Subj. 2008, 1780, 743-749. [CrossRef] [PubMed]

12. Vo, T.S.; Ngo, D.H.; Kim, S.K. Marine algae as a potential pharmaceutical source for anti-allergic therapeutics. Process Biochem. 2012, 47, 386-394. [CrossRef]

13. Yoshioka, H.; Ishida, M.; Nishi, K.; Oda, H.; Toyohara, H.; Sugahara, T. Studies on anti-allergic activity of Sargassum horneri extract. J. Funct. Foods 2014, 10, 154-160. [CrossRef]

14. Tanaka, T.; Shnimizu, M.; Moriwaki, H. Cancer chemoprevention by carotenoids. Molecules 2012, 17, 3202-3242. [CrossRef] [PubMed]

15. Heo, S.J.; Yoon, W.J.; Kim, K.N.; Ahn, G.N.; Kang, S.M.; Kang, D.H.; Oh, C.; Jung, W.K.; Jeon, Y.J. Evaluation of anti-inflammatory effect of fucoxanthin isolated from brown algae in lipopolysaccharide-stimulated RAW 264.7 macrophages. Food Chem. Toxicol. 2010, 48, 2045-2051. [CrossRef] [PubMed]

16. Zaragozá, M.; López, D.P.; Sáiz, M.; Poquet, M.; Pérez, J.; Puig-Parellada, P.; Marmol, F.; Simonetti, P.; Gardana, C.; Lerat, Y. Toxicity and antioxidant activity in vitro and in vivo of two Fucus vesiculosus extracts. J. Agric. Food Chem. 2008, 56, 7773-7780. [CrossRef] [PubMed] 
17. Beppu, F.; Niwano, Y.; Tsukui, T.; Hosokawa, M.; Miyashita, K. Single and repeated oral dose toxicity study of fucoxanthin (FX), a marine carotenoid, in mice. J. Toxicol. Sci. 2009, 34, 501-510. [CrossRef] [PubMed]

18. Beppu, F.; Niwano, Y.; Sato, E.; Kohno, M.; Tsukui, T.; Hosokawa, M.; Miyashita, K. In vitro and in vivo evaluation of mutagenicity of fucoxanthin (FX) and its metabolite fucoxanthinol (FXOH). J. Toxicol. Sci. 2009, 34, 693-698. [CrossRef] [PubMed]

19. Kanazawa, K.; Ozaki, Y.; Hashimoto, T.; Das, S.K.; Matsushita, S.; Hirano, M.; Okada, T.; Komoto, A.; Mori, N.; Nakatsuka, M. Commercial-scale preparation of biofunctional fucoxanthin from waste parts of brown sea algae Laminalia japonica. Food Sci. Technol. Res. 2008, 14, 573-582. [CrossRef]

20. Xiao, X.; Si, X.; Yuan, Z.; Xu, X.; Li, G. Isolation of fucoxanthin from edible brown algae by microwave-assisted extraction coupled with high-speed countercurrent chromatography. J. Sep. Sci. 2012, 35, 2313-2317. [CrossRef] [PubMed]

21. Mori, K.; Ooi, T.; Hiraoka, M.; Oka, N.; Hamada, H.; Tamura, M.; Kusumi, T. Fucoxanthin and its metabolites in edible brown algae cultivated in deep seawater. Mar. Drugs 2004, 2, 63-72. [CrossRef]

22. Jaswir, I.; Noviendri, D.; Salleh, H.M.; Miyashita, K. Fucoxanthin extractions of brown seaweeds and analysis of their lipid fraction in methanol. Food Sci. Technol. Res. 2012, 18, 251-257. [CrossRef]

23. Jaswir, I.; Noviendri, D.; Salleh, H.M.; Taher, M.; Miyashita, K.; Ramli, N. Analysis of fucoxanthin content and purification of all-trans-fucoxanthin from Turbinaria turbinata and Sargassum plagyophyllum by $\mathrm{SiO}_{2}$ open column chromatography and reversed phase-HPLC. J. Liq. Chromatogr. Relat. Technol. 2013, 36, 1340-1354. [CrossRef]

24. Fourest, E.; Volesky, B. Alginate properties and heavy metal biosorption by marine algae. Appl. Biochem. Biotechnol. 1997, 67, 215-226. [CrossRef]

25. Jeffryes, C.; Gutu, T.; Jiao, J.; Rorrer, G.L. Metabolic insertion of nanostructured $\mathrm{TiO}_{2}$ into the patterned biosilica of the diatom Pinnularia sp. by a two-stage bioreactor cultivation process. ACS Nano 2008, 2, 2103-2112. [CrossRef] [PubMed]

26. Wen, Z.Y.; Chen, F. A perfusion-cell bleeding culture strategy for enhancing the productivity of eicosapentaenoic acid by Nitzschia laevis. Appl. Microbiol. Biotechnol. 2001, 57, 316-322. [CrossRef] [PubMed]

27. Mooij, P.; van Loosdrecht, M.; Kleerebezem, R. Storage Compound Production by Phototrophic Diatoms; European Patent Office: Delft, The Nertherland, 2015.

28. Kim, S.M.; Kang, S.-W.; Kwon, O.-N.; Chung, D.; Pan, C.H. Fucoxanthin as a major carotenoid in Isochrysis aff. galbana: Characterization of extraction for commercial application. J. Korean Soc. Appl. Biol. Chem. 2012, 55, 477-483. [CrossRef]

29. Pasquet, V.; Chérouvrier, J.R.; Farhat, F.; Thiéry, V.; Piot, J.M.; Bérard, J.B.; Kaas, R.; Serive, B.; Patrice, T.; Cadoret, J.P. Study on the microalgal pigments extraction process: Performance of microwave assisted extraction. Process Biochem. 2011, 46, 59-67. [CrossRef]

30. Lewin, J.C. Heterotrophy in Diatoms. J. Gen. Microbiol. 1953, 9, 305-313. [CrossRef] [PubMed]

31. Wen, Z.-Y.; Chen, F. Production potential of eicosapentaenoic acid by the diatom Nitzschia laevis. Biotechnol. Lett. 2000, 22, 727-733. [CrossRef]

32. Stransky, H.; Hager, A. The carotenoid pattern and the occurrence of the light-induced xanthophyll cycle in various classes of algae. VI. Chemosystematic study. Arch. Mikrobiol. 1969, 73, 315-323. [CrossRef]

33. Kolber, Z.; Zehr, J.; Falkowski, P. Effects of growth irradiance and nitrogen limitation on photosynthetic energy conversion in photosystem II. Plant Physiol. 1988, 88, 923-929. [CrossRef] [PubMed]

34. Havaux, M.; Niyogi, K.K. The violaxanthin cycle protects plants from photooxidative damage by more than one mechanism. P. Natl. Acad. Sci. USA 1999, 96, 8762-8767. [CrossRef]

35. Reddy, C.; Jha, B.; Fujita, Y.; Ohno, M. Seaweed micropropagation techniques and their potentials: An overview. J. Appl. Phycol. 2008, 20, 609-617. [CrossRef]

36. Coesel, S.; Oborník, M.; Varela, J.; Falciatore, A.; Bowler, C. Evolutionary origins and functions of the carotenoid biosynthetic pathway in marine diatoms. PLoS ONE 2008, 3, e2896. [CrossRef] [PubMed]

37. Pahl, S.L.; Lewis, D.M.; Chen, F.; King, K.D. Heterotrophic growth and nutritional aspects of the diatom Cyclotella cryptica (Bacillariophyceae): Effect of some environmental factors. J. Biosci. Bioeng. 2010, 109, 235-239. [CrossRef] [PubMed]

38. Olaizola, M.; Roche, J.L.; Kolber, Z.; Falkowski, P.G. Non-photochemical fluorescence quenching and the diadinoxanthin cycle in a marine diatom. Photosynth. Res. 1994, 41, 357-370. [CrossRef] [PubMed] 
39. Lohr, M.; Wilhelm, C. Algae displaying the diadinoxanthin cycle also possess the violaxanthin cycle. Proc. Natl. Acad. Sci. USA 1999, 96, 8784-8789. [CrossRef] [PubMed]

40. Olaizola, M.; Yamamoto, H.Y. Short-term response of the diadinoxanthin cycle and fluorescence yield to high irradiance in Chaetoceros muelleri (Bacillariophyceae). J. Phycol. 1994, 30, 606-612. [CrossRef]

41. Piovan, A.; Seraglia, R.; Bresin, B.; Caniato, R.; Filippini, R. Fucoxanthin from Undaria pinnatifida: Photostability and coextractive effects. Molecules 2013, 18, 6298-6310. [CrossRef] [PubMed]

42. Xia, S.; Wang, K.; Wan, L.; Li, A.; Hu, Q.; Zhang, C. Production, characterization, and antioxidant activity of fucoxanthin from the marine diatom Odontella aurita. Mar. Drugs 2013, 11, 2667-2681. [CrossRef] [PubMed]

43. Foo, S.C.; Yusoff, F.M.; Ismail, M.; Basri, M.; Chan, K.W.; Khong, N.M.; Yau, S.K. Production of fucoxanthin-rich fraction (FxRF) from a diatom, Chaetoceros calcitrans (Paulsen) Takano 1968. Algal Res. 2015, 12, 26-32. [CrossRef]

44. Lewin, J.; Hellebust, J.A. Heterotrophic nutrition of the marine pennate diatom, Cylindrotheca fusiformis. Can. J. Microbiol. 1970, 16, 1123-1129. [CrossRef] [PubMed]

45. Mulders, K.J.; Weesepoel, Y.; Lamers, P.P.; Vincken, J.-P.; Martens, D.E.; Wijffels, R.H. Growth and pigment accumulation in nutrient-depleted Isochrysis aff. galbana T-ISO. J. Appl. Phycol. 2013, 25, 1421-1430. [CrossRef]

46. Roy, S.; Llewellyn, C.A.; Egeland, E.S.; Johnsen, G. Phytoplankton Pigments: Characterization, Chemotaxonomy and Applications in Oceanography; Cambridge University Press: Cambridge, UK, 2011.

47. Lewin, J.C.; Lewin, R.A. Auxotrophy and heterotrophy in marine littoral diatoms. Can. J. Microbiol. 1960, 6, 127-134. [CrossRef] [PubMed]

48. Wen, Z.; Chen, F. Heterotrophic production of eicosapentaenoid acid by the diatom Nitzschia laevis: Effects of silicate and glucose. J. Ind. Microbiol. Biotechnol. 2000, 25, 218-224. [CrossRef]

49. Li, Y.; Huang, J.; Sandmann, G.; Chen, F. Glucose sensing and the mitochondrial alternative pathway are involved in the regulation of astaxanthin biosynthesis in the dark-grown Chlorella zofingiensis (Chlorophyceae). Planta 2008, 228, 735-743. [CrossRef] [PubMed]

50. Syrett, P.; Wong, H.-A. The fermentation of glucose by Chlorella vulgaris. Biochem. J. 1963, 89, $308-315$. [CrossRef] [PubMed]

51. Tabernero, A.; del Valle, E.M.M.; Galán, M.A. Evaluating the industrial potential of biodiesel from a microalgae heterotrophic culture: Scale-up and economics. Biochem. Eng. J. 2012, 63, 104-115. [CrossRef]

52. Vazhappilly, R.; Chen, F. Heterotrophic production potential of omega-3 polyunsaturated fatty acids by microalgae and algae-like microorganisms. Bot. Mar. 1998, 41, 553-558. [CrossRef]

53. Pahl, S.L.; Lewis, D.M.; King, K.D.; Chen, F. Heterotrophic growth and nutritional aspects of the diatom Cyclotella cryptica (Bacillariophyceae): Effect of nitrogen source and concentration. J. Appl. Phycol. 2012, 24, 301-307. [CrossRef]

54. Arumugam, M.; Agarwal, A.; Arya, M.C.; Ahmed, Z. Influence of nitrogen sources on biomass productivity of microalgae Scenedesmus bijugatus. Bioresour. Technol. 2013, 131, 246-249. [CrossRef] [PubMed]

55. Wu, L.F.; Chen, P.C.; Lee, C.M. The effects of nitrogen sources and temperature on cell growth and lipid accumulation of microalgae. Int. Biodeterior. Biodegrad. 2013, 85, 506-510. [CrossRef]

56. Fidalgo, J.P.; Cid, A.; Torres, E.; Sukenik, A.; Herrero, C. Effects of nitrogen source and growth phase on proximate biochemical composition, lipid classes and fatty acid profile of the marine microalga Isochrysis galbana. Aquaculture 1998, 166, 105-116. [CrossRef]

57. DeBoer, J.A.; Guigli, H.J.; Israel, T.L.; D'Elia, C.F. Nutritional studies of two red algae. I. Growth rate as a function of nitrogen source and concentration. J. Phycol. 1978, 14, 261-266. [CrossRef]

58. Li, Y.; Horsman, M.; Wang, B.; Wu, N.; Lan, C.Q. Effects of nitrogen sources on cell growth and lipid accumulation of green alga Neochloris oleoabundans. Appl. Microbiol. Biotechnol. 2008, 81, 629-636. [CrossRef] [PubMed]

59. Alipanah, L.; Rohloff, J.; Winge, P.; Bones, A.M.; Brembu, T. Whole-cell response to nitrogen deprivation in the diatom Phaeodactylum tricornutum. J. Exp. Bot. 2015, 66, 6281-6296. [CrossRef] [PubMed]

60. Willemoës, M.; Monas, E. Relationship between growth irradiance and the xanthophyll cycle pool in the diatom Nitzschia palea. Physiol. Plant. 1991, 83, 449-456. [CrossRef]

61. Van de Poll, W.H.; van Leeuwe, M.A.; Roggeveld, J.; Buma, A.G. Nutrient limitation and high irradiance acclimation reduce PAR and UV-induced viability loss in the antarctic diatom Chaetoceros brevis (Bacillariophyceae). J. Phycol. 2005, 41, 840-850. [CrossRef] 
62. Gómez-Loredo, A.; Benavides, J.; Rito-Palomares, M. Growth kinetics and fucoxanthin production of Phaeodactylum tricornutum and Isochrysis galbana cultures at different light and agitation conditions. J. Appl. Phycol. 2015, 1-12. [CrossRef]

63. Chen, T.; Liu, J.; Guo, B.; Ma, X.; Sun, P.; Liu, B.; Chen, F. Light attenuates lipid accumulation while enhancing cell proliferation and starch synthesis in the glucose-fed oleaginous microalga Chlorella zofingiensis. Sci. Rep. 2015, 5. [CrossRef] [PubMed]

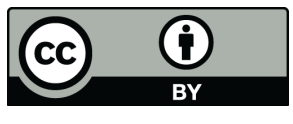

(C) 2016 by the authors; licensee MDPI, Basel, Switzerland. This article is an open access article distributed under the terms and conditions of the Creative Commons Attribution (CC-BY) license (http://creativecommons.org/licenses/by/4.0/). 